\title{
Protée
}

\section{Lumières, matières et paysages}

\section{Jacques Fontanille}

Volume 31, numéro 3, hiver 2003

\section{Lumières}

URI : https://id.erudit.org/iderudit/008434ar

DOI : https://doi.org/10.7202/008434ar

Aller au sommaire du numéro

\section{Éditeur(s)}

Département des arts et lettres - Université du Québec à Chicoutimi

\section{ISSN}

0300-3523 (imprimé)

1708-2307 (numérique)

Découvrir la revue

\section{Citer cet article}

Fontanille, J. (2003). Lumières, matières et paysages. Protée, 31(3), 17-30.

https://doi.org/10.7202/008434ar

\section{Résumé de l'article}

Parmi tous les effets de lumière, on a choisi de prêter attention à l'un d'eux, car il a trait aux rapports entre le temps et la matière : il s'agit de la " lumière intérieure » dans le paysage. La lumière interne devient alors une propriété des objets et de leur structure matérielle, en plusieurs directions : l'animation actantielle, puisque l'intensité de l'éclat émane de l'objet ; la modalité et l'aspectualité, puisque la matière module la " restitution » de cet éclat ; la temporalité, enfin, puisque la perception de ces lumières est alors dissociée de celle de la source et du moment de l'émission. Ces remarques générales sont exploitées au cours d'une analyse des Fleurs du Mal, de Baudelaire, où on examine notamment le " feu du regard ", la " lumière voilée " et le " soleil lavé ». Elles sont ensuite étendues à une réflexion sur le "paysage ", conçu comme configuration sémiotique émanant du monde naturel, à la rencontre entre une " existence " et une " expérience ".
Ce document est protégé par la loi sur le droit d'auteur. L'utilisation des services d'Érudit (y compris la reproduction) est assujettie à sa politique d'utilisation que vous pouvez consulter en ligne.

https://apropos.erudit.org/fr/usagers/politique-dutilisation/ 


\section{LU M IÈRES, M ATIÈRES ET PAYSAG ES}

JACQUES FONTANILLE

\section{INTRODUCTION}

Parmi tous les effets de lumière, il en est un qui mérite toute l'attention du sémioticien, car il a trait aux rapports entre le temps et la matière: à côté en effet de l'éclairage, de la lumière extérieure, voire de la lumière associée aux couleurs, la "lumière intérieure» forme un motif à part, qui est tout particulièrement exploité, dans certaines installations ou expositions, par exemple, lorsque les œuvres sont éclairées par derrière.

La lumière interne devient alors une propriété des objets, soit de leur structure matérielle, soit de leur enveloppe ou de leur surface, et cette propriété sollicite l'interprétation en plusieurs sens: (i) du côté de l'animation actantielle, puisque l'intensité de l'éclat émane de l'objet; (ii) du côté de la modalité et de l'aspectualité, puisque la matière module la «restitution» de cet éclat et peut du même coup en infléchir, voire en convertir une partie des propriétés sémantiques et syntaxiques; (iii) du côté de la temporalité, enfin, puisque la perception de ces lumières est alors dissociée de celle de la source et du moment de l'émission: le corps matériel apparaît à cet égard comme un médiateur temporalisant, l'actant de contrôle entre la source et la cible.

Toutes ces voies de l'interprétation étant rassemblées, elles se constituent en configurations passionnelles, des avatars pathémiques de la matière-temps.

Ces remarques générales seront exploitées au cours d'une réflexion sur le "paysage», conçu comme configuration sémiotique émanant du monde naturel. Mais nous commencerons pourtant par l'étude d'un texte littéraire, le recueil Les Fleurs du Mal de Baudelaire, qui nous permettra de déployer l'ensemble du dispositif de l'analyse.

\section{LA LUMIĖRE ET LE PAYSAGE CHEZ BAUDELAIRE}

Dans un ouvrage maintenant introuvable, Claude Zilberberg avait montré, à propos des Fleurs du Mal, le rôle joué par la figure composite "soleil + liquide», notamment dans la distribution des rôles «émetteur» et «récepteur» dans un processus de communication qui se déploie à l'intérieur du monde naturel (Zilberberg, 1972). 
Nous voudrions reprendre cette question, mais sous un angle un peu différent, puisqu'il s'agit de comprendre quelles sont les propriétés sémantiques et syntaxiques d'une «lumière intérieure du paysage»; et, pour cela, être particulièrement attentif, pour chaque type de figure, d'une part au mode de saisie qu'il induit et, d'autre part, à ses effets axiologiques. Mais l'«intériorité» dont il est question ici, s'agissant d'une portion du monde naturel, est toute matérielle: la description vise donc plus particulièrement la manière dont la lumière s'associe aux matières, et notamment aux liquides, par mélange, par reflet, par transition, etc.

\section{LUMIĖRE, REGARD, YEUX DE FEU}

Le cas le plus simple de la lumière saisie comme émission à partir d'une intériorité est celui des «yeux de feu ", figure récurrente dans le recueil, qui est systématiquement traitée comme euphorique, mais une euphorie qui serait en quelque sorte "figée", puisque sans effet sensible sur le corps du sujet récepteur, à l'exception de l'enveloppe lumineuse dont il l'habille. Mais cette «émanation» est une émanation transcendante, et elle s'inscrit fréquemment sur une isotopie "spirituelle» («mystique» ou "métaphysique»); en outre, elle partage ces deux propriétés avec le parfum, et notamment celui de la chair vivante et du sang. Ainsi, dans Spleen XLII ${ }^{1}$

- Nous mettrons notre orgueil à chanter ses louanges:

Rien ne vaut la douceur de son autorité;

Sa chair spirituelle a le parfum des Anges

Et son œil nous revêt d'un habit de clarté. (p.55)

Le parfum est à la chair ce que la lumière est au regard, et les deux ont une dimension "spirituelle». On voit que, dans ce cas, l'appréhension est cognitive, bien que le vecteur de la communication soit sensoriel: dans ce cas, la synesthésie est purement rhétorique, obéissant à un système semi-symbolique qui conduit à une équivalence grâce à un raisonnement proportionnel implicite; [Le parfum est à la chair ce que la lumière est au regard] a en effet la forme de la proportion

d'Aristote.

En outre, cette "passivation sensible" prend la forme d'un enveloppement: le parfum enveloppe par définition le corps percevant (Fontanille, 1999: 34-37), de la même manière que, plus explicitement, le regard l'«habille» de lumière. L'enveloppe émise et projetée par la chair-source se substitue alors à l'enveloppe cœnesthésique du corps sensible; on notera tout particulièrement le choix de modes sensoriels «passivants». En effet, l'odeur émise, par quelque corps que ce soit, enveloppe les autres corps contre leur gré; c'est un fait souligné par tous les commentateurs (Kant, [1798] 1979: 40), l'odeur est un pouvoir-faire qui rencontre ou suscite, du côté du corps percevant, un ne pas pouvoir ne pas faire (Fontanille, 1999: 53-54).

Pour la vision, c'est l'inversequi se produit: le regard du corps percevant se saisit des autres corps et leur reconnaît une enveloppe "débrayée", une limite propre entre un intérieur et un extérieur; grâce à un déplacement modal, du savoir et croire au pouvoir et vouloir, la "reconnaissance» d'une enveloppe primaire (propre) est convertie en "projection" d'une enveloppe secondaire (non propre), et c'est ainsi qu'on obtient: Et son œil nous revêt d'un habit de clarté; dès lors, c'est le corps perçu (par le regard) qui est sous le régime du ne pas pouvoir ne pas faire. Mais ce corps "perçu» est encore un corps percevant (il voit qu'on le regarde), et c'est très exactement de son point de vue que se produit l'enveloppement; par conséquent, même si la situation est un peu plus complexe que pour l'odeur, le résultat modal et figuratif est globalement identique.

La réponse du sujet perçu-percevant sera alors doublement infléchie par les traits «cognitif» et «transcendant»: dans Spleen XLII, c'est en effet une «louange» adressée à la beauté, considérée comme une entité d'essence supérieure. La passivation s'accompagne donc d'une capture du corps percevant, et l'ensemble du processus perd tout caractère d' "animation ", comme dans Spleen et Idéal LI (Le chat II).

Je vois avec étonnement

Le feu de tes prunelles pâles,

Clairs fanaux, vivantes opales,

Qui me contemplent fixement. (p.64)

Le principe qui sous-tend l'ensemble de ces propriétés (passivation, capture, enveloppement, 
figement) pourrait maintenant être suggéré: la «douceur» qui, dans le premier poème était le ressort de l'«autorité», et, qui, dans le second, est tout simplement celui de l'efficience: «si doux... que... je fus embaumé». La douceur suppose un ralentissement, un tempo presque arrêté, à peine perceptible (et qui conforterait l'effet de figement), elle est pourtant d'une efficience instantanée (rien qu'une fois, étonnement); d'un côté, elle est définie par un parcours figuratif réglé par l'atténuation de l'intensité et le ralentissement du tempo, qui pérennise un contact minimal, et, de l'autre côté, elle reçoit, dans ce texte en particulier, une valeur modale de pouvoir faire intense et immédiat.

Par ailleurs, si nous revenons à la source de toute syntaxe figurative, c'est-à-dire aux interactions entre matière et énergie (Fontanille, 2003), la lumière en tant que force traverse des matières dont elle émane; selon le cas et en général, ces matières amplifient ou atténuent l'intensité de la force, ce qui revient, à un autre niveau, à en magnifier ou ravaler la valeur. Mais l'interaction entre matière et énergie agit sur d'autres paramètres: sans en affaiblir l'intensité, et sans en ravaler la valeur, la matière peut moduler le tempo de restitution de la lumière, et convertir ainsi l'efficacité attachée à l'intensité en une efficience indirecte, induite par la lenteur: l'intensité, en effet, ne peut être que subie et reçue, alors que la douceur et la lenteur peuvent être partagées, ce qui constitue un changement dans le procédé de manipulation sensible. En somme, l'action de la matière sur la lumière garantit l'acceptabilité de cette lumière par le corps perçu-percevant, en facilite la réception et engage l'interaction communicative avec les corps-actants.

C'est donc le tempo de l'émission (de la lumière comme du parfum) réglé par la matière émettrice ellemême (œil ou fourrure) qui induit le figement, l'enveloppement et la capture par synchronisation: c'est très précisément ce processus que nous pouvons caractériser comme «une manipulation sensible et figurative».

Il y a alors communication de quelque chose qui dépasse le sujet, et qui pourtant semble se dégager d'une matière et d'un lieu identifiable, comme si cette matière et ce lieu faisaient fonction de médiateurs pour le transit et la modulation d'un flux sensible émanant d'un "ailleurs" insaisissable.

En somme, à ce stade de l'analyse, les propriétés pertinentes seraient les suivantes:

- une figure propre à un mode sensoriel (la lumière) s'associe avec des matières (eau ou autre);

- la nature de cette association détermine une esthésie particulière et une projection thymique spécifique (positive ou négative);

- cette configuration accueille de manière préférentielle telle ou telle synesthésie (avec l'odeur, le bruit ou le goût).

En somme, les associations polysensorielles seraient déterminées par des projections thymiques qui caractériseraient des interactions spécifiques entre énergies (ici, la lumière) et matières (ici, l'eau pour l'essentiel).

\section{SOLEIL, LUMIÈRE ET VAPEUR}

Le rapport entre lumière, regard et matière n'a pas été entièrement établi jusqu'ici; à cet égard, les occurrences utiles dans Les Fleurs du Mal sont rares, mais elles apparaissent dans le contexte d'un rapprochement avec une figure remarquable (et tout particulièrement remarquée par Claude Zilberberg dans l'étude déjà évoquée) : le "soleil mouillé». En voici un exemple, dans L'Invitation au voyage:
Mon enfant, ma sœur,
Songe à la douceur
D'aller là-bas viure ensemble!
[...]
Les soleils mouillés
De ces ciels brouillés
Pour mon esprit ont les charmes
Si mystérieux
De tes traîtres yeux,
Brillants à travers leurs larmes. [...]
- Les soleils couchants
Revêtent les champs [...]
Le monde s'endort
Dans une chaude lumière.

(Spleen et Idéal LIII, p.66-67) 
Tout d'abord, on y rencontre une partie des propriétés reconnues à la configuration précédente: d'un côté, l'ensemble de l'interaction est sous le régime de la douceur et de la lenteur, notamment dans le refrain (l'"ordre» et le "calme»), et, de l'autre, la lumière émise induit à la fois l'enveloppement et le figement du corps perçu-percevant (qui est ici «le monde» lui-même: les champs revêtus de soleil); l'endormissement résume à lui seul ces propriétés, tout en portant en même temps la même explication: une efficience de la douceur lumineuse, qui impose son tempo au paysage, par enveloppement («dans») et ralentissement synchrone.

Mais, pour ce qui concerne le regard, il est cette fois lui-même soumis, tout comme le soleil, à une condition matérielle: le liquide, le «mouillé». Les larmes sont aux yeux ce que les vapeurs brumeuses sont au soleil: une condition d'efficience. Et cette condition suppose que la lumière soit convertie en matière mouillée qui enrobe les figures.

Pourtant, il est impossible de rabattre entièrement cette configuration sur la première: dans la précédente, en effet, la lumière était transcendante, elle se saisissait du corps perçu-percevant pour lui communiquer des valeurs supérieures, et avec une évaluationglobalement euphorique; cette même lumière, sans être directement matérialisée dans le regard, était systématiquement associée à la chair et au parfum, détenteur de la même transcendance. Ici, au contraire, la lumière mouillée est immanente et ambivalente, elle n'est plus associée à l'olfaction.

Elle est immanente car elle ne donne accès à aucun destinateur; elle appartient elle aussi à un "ailleurs", mais qui est explicitement déterminé: c'est un «là-bas», certes utopique, mais défini comme le lieu projeté au terme d'un voyage, alors que l'«ailleurs» de la précédente lumière était seulement implicite et présupposé. Elle aussi donne à connaître ou à deviner, mais de manière immanente: en effet, le mystère qui est ici évoqué est une figure cognitive qui, à la différence de l'occulte, se donne comme une manifestation indirecte et incomplète d'un secret; quelque chose se donne à sentir, qui est la présence d'un objet cognitif, et dont le contenu peut indifféremment être appréhendé ou rester inaccessible. Mais, étant de l'ordre de la présence immédiate, le mystère est immanent à la chose même et à sa substance matérielle; il l'est d'autant plus que, considéré comme un mode de l'appréhension d'une figure sensible, il ne parvient ni à la stabiliser ni à l'identifier: la saisie mystérieuse est au moins une saisie anté-iconique.

Cette structure sensible et véridictoire reçoit dans le texte une expression figurative remarquable: que ce soient les yeux ou le soleil, ils sont non pas imprégnés d'eau ou plongés dans une masse liquide; ils sont seulement voilés: l'eau est à la fois un masque et un filtre, qui laisse passer l'intensité lumineuse de la présence, sans laisser voir le contenu de cette présence (par exemple, dans le cas des yeux, la "vérité» de l'état d'âme actuel) ${ }^{2}$.

En outre, l'effet du mystère n'est pas, comme précédemment, une capture immédiate (l'étonnement, par exemple, ou l'embaumement), mais un charme. Le charme est une action magique incarnée: il n'y a de charme qu'entre des corps, ou par l'intermédiaire des corps. Le retour à l'immanence s'accompagne par conséquent d'une réactivation du corps sensible (présence, mystère, charme ne peuvent se comprendre sans la participation de la sensibilité). D'un point de vue sémiotique, le charme serait ici le signifiant du mystère; comme ces figures sont produites par une enveloppe d'eau filtrante, alors on peut considérer que le charme est le mode d'inscription du signifiant sur l'enveloppe du regard et de la source de lumière.

La lumière mouillée est en outre ambivalente, car elle est à la fois attirante et inquiétante: le mystère, en effet, provoque le trouble du corps sensible, car cette morphologie qui résiste à l'iconisation, qui se dérobe et qui ne peut être appréhendée autrement qu'instable, affecte le corps sensible de la même instabilité. Si l'on associe la dérobade de l'objet (le secret, présentifié mais sans son contenu), le filtre et l'inscription du charme, et le trouble du sujet, alors on comprend pourquoi les yeux peuvent être «traitres» et inquiétants: l'ensemble de ce nouveau dispositif sensible et figuratif, dans ce cas, loin de délivrer la clé d'une transcendance éthico-esthétique de type cognitif, permet aux interactions communicatives de se développer en stratégies et contre-stratégies, en 
dissimulations, distorsions, cryptage et décryptage des signifiants figuratifs. Mais, plus généralement, c'est cette forme matérielle même de la lumière qui porte une axiologie ambivalente: splendeur et danger (l'attirance sensorielle de l'Autre et la menace pour l'intégrité du Moi), comme en témoigne Ciel brouillé:

Tu ressembles parfois à ces beaux horizons

Qu'allument les soleils des brumeuses saisons...

Comme tu resplendis, paysage mouillé

Qu'enflamment les rayons tombant d'un ciel brouillé!

Ô fermme dangereuse, ô séduisants climats!

(Spleen et Idéal L, p.62)

Entre L'Invitation au voyage et Ciel brouillé, le point de vue s'est inversé: dans le premier, on adoptait le point de vue de la source (le regard voilé de larmes, le soleil mouillé); dans le second, on adopte strictement le point de vue de la cible, le corps perçu-percevant. La splendeur et le danger sont donc des effets de sens qui, du côté de la cible, répondent au mystère et, du côté de la source, au charme. Comme on pouvait dire tout à l'heure que le charme est le signifiant de manifestation du mystère, la splendeur est le signifiant de manifestation du danger. Le dispositif syntaxique précédent peut donc être maintenant présenté comme un syntagme d'interaction sémiotique:

\begin{tabular}{|cccc|}
\hline & SOURCE & CONTRÔLE & CIBLE \\
Signifiant & Charme & Voile (transit et filtre) & Splendeur \\
SignifiÉ & Mystère & Tri véridictoire & Danger \\
\hline
\end{tabular}

\section{LE SOLEIL LAVÉ DANS LA MER}

Les rapports de la lumière et de l'eau donnent lieu à une dernière figure. En effet, le soleil et la lumière peuvent être mouillés par immersion. Il n'y a plus alors diffusion et médiation, mais «rajeunissement», restauration axiologique, renouveau de la lumière: la mer et les masses liquides font alors office non de diffuseur, mais de lieu critique pour la réactualisation des valeurs: elles trient et elles restaurent les valeurs...

Les lumières mouillées diffuses et présentes étaient ambivalentes; les lumières intermittentes et distantes étaient simplement dysphoriques; les lumières liquides, ou purifiées par la mer, sont simplement euphoriques.
Dans Élévation, par exemple, «[1]e feu clair qui remplit les espaces limpides» est une "pure et divine liqueur», et s'oppose alors radicalement à «l'existence brumeuse " (Spleen et Idéal III, p. 25). Mais c'est dans La Vie antérieure que la transfiguration est la plus spectaculaire:

J'ai longtemps habité sous de vastes portiques

Que les soleils marins teintaient de mille feux, [...]

Les houles, en roulant les images des cieux,

Mêlaient d'une façon solennelle et mystique

Les tout-puissants accords de leur riche musique

Aux couleurs du couchant reflété par mes yeux.

(Spleen et Idéal XII, p.28)

Là aussi, la mer («les houles») et le soleil («les couleurs du couchant») célèbrent ensemble un rite («une façon solennelle et mystique»), mais sous des conditions bien différentes. Tout d'abord, nous n'avons plus seulement deux actants (un émetteur et un récepteur), mais trois (la mer, le soleil, «mes yeux»), qui sont tour à tour émetteurs et récepteurs; ensuite, la relation entre les trois actants a changé: il n'est plus question ni de mélange, ni de diffusion, ni de voile, mais uniquement de reflets: les soleils "teintent» les portiques, les houles «roulent des images des cieux» et le couchant est «reflété» par les yeux de l'observateur. En outre, le mouvement a remplacé le figement et la lenteur. Enfin, les sensations associées ne sont pas olfactives mais auditives ("les accords de leur riche musique»): autre dispositif matière/énergie, autre projection thymique, et donc: autre synesthésie.

Le mouvement généralisé ainsi que la modification de la structure actantielle font de cette figure lumineuse une figure d'activation généralisée: le premier dispositif comportait une relation, entre source et cible, de type passivant et sans instance de contrôle; le second (ci-dessus) est à la fois passivant (fascinant) et avec actant de contrôle (le voile); le dernier, celui-ci, est activant et comporte un actant de contrôle: la source est toujours le soleil, mais il a deux cibles, la mer et les yeux, chacune d'elles fonctionnant tout à tour comme actant de contrôle quand l'autre est la cible; mais, en outre, les relations source/cible/ contrôle s'inversent, grâce au reflet: quand la mer ou les yeux reflètent le soleil, ils deviennent la source 
d'une image, qu'ils se renvoient l'un à l'autre, et que contrôle le soleil. Ce dispositif généralise le principe d'un échange permanent, distribué et généralisé.

Le soleil est reflété deux fois: une fois par la mer, une fois par les yeux, ce qui implique que l'observateur s'en procure deux images, une directe et une indirecte. La question ne se pose donc plus en termes de synchronisation des tempos, mais en termes de superposition et d'accord instantané entre les imagesempreintes, exactement comme dans un accord musical; c'est pourquoi la vision et la lumière dialoguent ici avec l'audition, et non plus avec l'olfaction.

\section{Enveloppes, filtres et membranes axiologiques}

Eu égard aux variétés fonctionnelles des enveloppes, pour commencer, on doit constater ici que la surface des cibles (portiques, houles, yeux) est devenue la surface d'inscription des signifiants de la lumière (les images). Nous avons déjà rencontré de telles inscriptions, mais, d'une part, il s'agissait de l'enveloppe des sources (regard ou soleil mouillés) et, d'autre part, l'eau n'est plus ici la matière d'une enveloppe filtrante, mais un corps lui-même doté d'une enveloppe.

Tout se passe comme si, partant d'une simple membrane interface, entre un intérieur et un extérieur, nous étions passés à l'intérieur de la membrane ${ }^{3}$ : celle-ci est alors elle-même traitée comme un corps, doté lui-même d'une structure matérielle et de sa propre enveloppe superficielle; la structure matérielle de la masse aquatique fonctionne comme le filtre de restauration des valeurs lumineuses, et l'enveloppe superficielle de cette masse, comme la surface d'inscription des signifiants-images.

En somme, la lumière et l'eau sont tour à tour enveloppantes et enveloppées: la lumière est enveloppante à l'égard des corps qu'elle entoure, mais elle est enveloppée par les voiles d'eau et de larmes; l'eau est à la fois enveloppante à l'égard des sources de lumière qu'elle voile, et enveloppée par les images-reflets du soleil. Et c'est très précisément cette dialectique des relations d'enveloppement entre la lumière et l'eau qui engendre la diversité des signifiants dans l'interaction communicative:
- douceur et lenteur: la lumière est enveloppante, l'eau est absente;

- charme et mystère: la lumière est enveloppée, l'eau est enveloppante;

- reflets et images: la lumière est enveloppante, l'eau est enveloppée.

En outre, l'interaction entre la lumière, l'eau et les corps obéit à plusieurs types de tempos et de régimes temporels différents et déterminés, correspondant très précisément chacun à une des variétés fonctionnelles de l'enveloppe en tant qu'opérateur: la lenteur, le trouble, le cycle et l'instant.

(i) La lenteur est celle de l'enveloppement immobilisant (l'enveloppe de contenance et de maintenance);

(ii) le trouble est celui provoqué par la traversée indécise de la lumière dans le voile humide (l'enveloppe comme filtre);

(iii) le cycle est celui de la régénération; c'est en général celui de la journée, entre soleil levant et soleil couchant (enveloppe de régénération);

(iv) l'instant, enfin, est celui de l'image (enveloppe comme surface d'inscription).

Chaque régime temporel est déterminé par une morphologie de l'interaction et du transfert entre source et cible, eux-mêmes conditionnés par les propriétés matérielles du contrôle (s'agissant des morphologies du transfert entre source et cible, nous pourrions y reconnaître en effet des variétés de l'actant de contrôle):

(i) la lenteur est déterminée par les contours d'un corps ou d'un monde qu'il faut suivre progressivement et en continuité, sans heurtset sans hiatus;

(ii) le trouble, par le caractère translucide de l'obstacle: ni opaque, ni transparent, il retient, pour un laps de temps indéfini, une partie indéterminée et non identifiable de la lumière qui le traverse;

(iii) le cycle, par la révolution de la terre autour du soleil, par le rythme de la vie du monde et des êtres;

(iv) l'instant, enfin, par le caractère impénétrable de l'obstacle, et sa capacité réfléchissante.

- Quatre régimes temporels pour

- quatre variétés fonctionnelles de l'enveloppe, et selon 
- quatre types morphologiques de l'interaction:

voilà en somme le système sémiotique des alliances de la lumière et de l'eau dans Les Fleurs du Mal. Pour en systématiser l'interdéfinition, nous pouvons partir de la structure des variétés fonctionnelles de l'enveloppe.

Les fonctions de maintenance et de contenance forment un ensemble homogène, qui s'oppose, sur l'axe de la contrariété, à la fonction de surface d'inscription: dans un cas, les signifiants sont «contenus-maintenus» à l'intérieur, dans l'autre, ils sont «affichés» et inscrits vers l'extérieur.

D'un côté, la fonction de filtre et de masque assure la transition entre la première et la deuxième positions: elle suspend la contenance et la maintenance, puisqu'elle laisse passer une partie des signifiants, en vue de leur inscription éventuelle sur la surface extérieure; de l'autre côté, la fonction de régénération suspend la fonction de surface d'inscription, car elle utilise des sollicitations extérieures pour les exploiter en vue d'une revalorisation de l'intérieur et, du même coup, elle prépare la réactualisation des fonctions de maintenance et de contenance. Ce sont donc les deux contradictoires, qui forment les subcontraires de la catégorie. On obtient donc le dispositif suivant, pour les VARIÉTÉS DE L'ENVELOPPE.

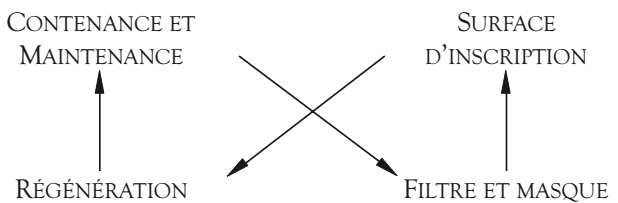

Cette structure nous permet de redistribuer et d'homologuer les modes d'interaction entre la lumière et l'eau, sous les espèces (i) des régimes temporels et (ii) des morphologies de l'interaction et du contrôle, (iii) avec les VARIÉTÉS DE L'ENVELOPPE:

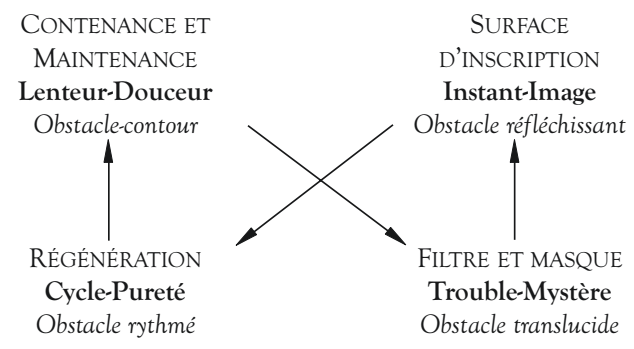

Dans le modèle ainsi décliné, on peut considérer, comme hypothèse de travail, que les trois composantes prises séparément sont généralisables à l'analyse des dispositifs figuratifs de la lumière dans le paysage, ainsi que le principe d'une homologation entre eux. En revanche, cette homologation-là, qui fait par exemple correspondre le régime temporel de l'instant à la fonction «surface d'inscription", ou celui du trouble à la fonction «filtre et masque», est spécifique du corpus étudié jusqu'ici, celui des Fleurs du Mal. Rien n'interdit d'imaginer d'autres homologations, dans d'autres corpus, comme celle qui ferait correspondre le régime temporel de la lenteurdouceur avec la fonction de surface d'inscription.

Transitions, conversions et constitution sémiotique $d u$ paysage: le cas du Balcon

Nous pouvons observer, pour finir, la transition entre ces deux états dans Le Balcon. La deuxième strophe commence par l'évocation d'un souvenir, sous la forme du «soleil mouillé» (opérateur: filtre):

Les soirs illuminés par l'ardeur $d u$ charbon,

Et les soirs au balcon, voilés de vapeurs roses.

(Spleen et Idéal XXXVI, p.48)

Cette évocation est ponctuée par deux autres appels à la lumière; le premier confirme l'énergie lumineuse, enveloppée dans la pénombre:

Que les soleils sont beaux dans les chaudes soirées! (ibid.)

Et le second note une modification matérielle de l'enveloppe (opérateur: surface extérieure):

La nuit s'épaississait ainsi qu'une cloison, [...] (ibid.)

À chaque apparition de la figure lumineuse correspond une sensation amoureuse:

- Que ton sein m'était doux, pour la première (la lumière voilée et filtrée) ;

- Je croyais respirer le parfum de ton sang, pour la deuxième (la lumière enveloppée);

- Je buvais ton souffle, pour la troisième (la lumière négative durcie en cloison).

Enfin, dans la dernière strophe, la figure solaire revient, prise en charge par une question, et sous la 
forme du «soleil lavé et rajeuni» (opérateur:

régénération):

Ces serments, ces parfums, ces baiser infinis,

Renaîtront-ils d'un gouffre interdit à nos sondes

Comme montent au ciel les soleils rajeunis

Après s'être lavés au fond des mers profondes? (p.49)

Dans le même poème, on assiste à la transition d'un état de la configuration à l'autre: on commence par les lumières mouillées, vaporeuses et diffuses, et on termine sur les lumières rajeunies par les mers. Entre les deux, le mouvement ralentit peu à peu, les amants s'endorment, et la nuit s'épaissit, pour former une surface protectrice dotée d'un intérieur et d'un extérieur: on assiste ainsi à une solidification progressive de l'enveloppe de la lumière, jusqu'à former une surface de séparation, de contenance et de maintenance (la "cloison»). L'enveloppe filtrante est devenue alors une frontière durable et tournée vers l'extérieur, qui pourrait recevoir d'éventuelles empreintes, mais qui ne fait que séparer et contenir durablement dans la lenteur et la douceur de l'endormissement.

Ce parcours correspond, dans le modèle proposé, aux positions suivantes, à ceci près que le parcours canonique est pris à rebours:

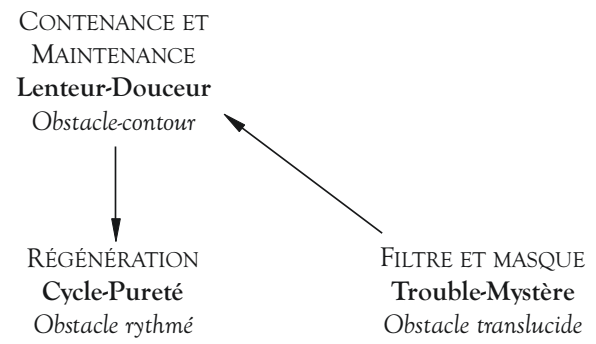

Cette inversion du parcours pourrait constituer un argument en faveur d'une inversion des positions entre contraires et subcontraires, qui conférerait au modèle la disposition proposée à la colonne de droite.

En l'état, le corpus ne permet pas de trancher entre ces deux dispositions; mais, comme dans l'une et l'autre, les positions axiologiques (euphorie et dysphorie) sont les mêmes, la différence est purement technique et finalement d'intérêt secondaire.

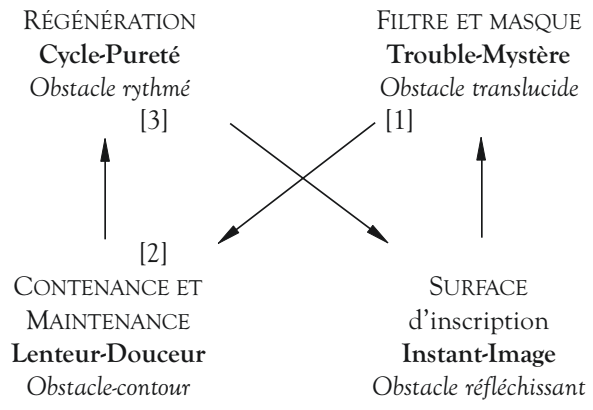

Par ailleurs, entre les positions initiales et terminales, dans le poème, la lumière a été associée à d'autres sensations, ce qui semble un effet systématique de sa "matérialisation»: le toucher et la caresse, le parfum, l'absorption. Mais si le toucher est bien procuré par la peau (le «sein»), il n'en est pas de même pour les autres: le parfum est celui du «sang», la boisson est le «souffle». Toutes ces alliances polysensorielles nous parlent en somme de ce qui émane du corps aimé: cette émanation n'est jamais redondante ou directe, elle forme chaque fois, pour l'amant, une «icône» sensorielle quasi indépendante de la partie corporelle qui l'a produite, selon un principe qui, d'une certaine manière, croise les éléments matériels et les vecteurs sensoriels:

(i) de la peau et du sein vont naître, pour l'amant, des paroles et des sermentsqu'il entend;

(ii) du cœur et du sang de l'aimée, un parfum qu'il respire;

(iii) de son souffle, une boisson qu'il absorbe.

C'est alors qu'on constate que le principe d'une élaboration sémiotique secondaire se confirme et que nous assistons à la production de signes et de valeurs à l'intérieur des interactions entre lumière et matière. Des signifiés corporels constitutifs de l'identité de l'autre, et plus précisément de sa chair vivante et motrice (toucher, circulation sanguine, respiration), vont se manifester pour le corps percevant du sujet comme des signifiants incorporables (qui peuvent être absorbés par le corps propre): paroles et figures auditives, parfum et figures olfactives, boisson et figures d'ingestion. La communication figurative devient alors une communication proprioceptive, les signifiés sensorimoteurs du corps autre étant convertis en signifiants d'absorption pour le corps propre. En somme, les propriétés «existentielles» du corps de l'autre sont 
converties en propriétés d'expérience pour le corps percevant. Globalement, un processus de constitution sémiotique du paysage se met donc en place au cours de ce poème:

(i) PHASE 1: APPRÉHENSION Lors d'une première appréhension, les icônes sensorielles se donnent à saisir comme des «mélanges» ou des associations entre matière et énergie (par exemple, le soleil voilé ou mouillé, ou le sang et l'odeur).

(ii) PhASe 2: SAISIE

Mais cette alliance étant reproduite plusieurs fois, elle est ensuite saisie comme une émanation indirecte de la sensation à partir des éléments matériels.

(iii) PHASE 3: COMPRÉHENSION

Enfin, elle se laisse comprendre comme une règle de conversion entre les signifiés issus du corps du paysage et les signifiants pour le corps de l'observateur.

C'est ainsi, qu'au début, la première apparition du soleil voilé est une simple association entre la lumière et l'eau, puis, dans les phases intermédiaires, d'autres sensations sont saisies comme émanations indirectes de cette association, et, enfin, dans la dernière strophe, la question finale pose la règle de conversion: le tri, la restauration de l'énergie (le soleil) par son passage dans une matière hétérogène (la mer).

Mais cette syntaxe figurative est aussi temporelle, et implique une mémoire:

Ces serments, ces parfums, ces baisers infinis,

Renaîtrontils d'un gouffre interdit à nos sondes

Comme montent au ciel les soleils rajeunis

Après s'être lavés au fond des mers profondes? (p.49)

Lors de la dernière phase, en effet, la renaissance est évoquée au futur, et le passage du soleil dans la mer lui est antérieur: le souvenir naît donc de la conversion des figures sensorielles, et la «lumière communicante» est alors porteuse de mémoire. Mais la résurgence du souvenir présuppose elle-même deux autres opérations: (i) l'enfouissement d'une expérience comme «empreinte» dans la matière corporelle, et (ii) le désenfouissement de cette expérience par une relecture de l'empreinte.

L'enfouissement a pour condition une certaine qualité de la structure matérielle de la surface d'inscription $^{4}$ : le voile d'eau durcie remplira cet office. Et le désenfouissement suppose, en outre, une modification de cette enveloppe, un «révélateur» ultérieur des inscriptions: la mer, en lavant les soleils, assurera cette régénération des empreintes-souvenirs.

Cela implique, de plus, que cette syntaxe figurative peut être interprétée de manière cohérente, grâce au parcours des différentes variétés fonctionnelles des «enveloppes» sémiotiques des corps:

(i) lors de la première APPRÉHENSION, l'enveloppe se donne à saisir comme appartenant à un élément matériel différent de celui dont émane l'énergie: elle est alors appréhendée comme une enveloppe de maintenance et de contenance;

(ii) mais elle est immédiatement saisie comme un "voile» et, lors de la reproduction et de la STABILISATION du phénomène, l'enveloppe est globalement comprise comme un filtre pour des émanations (tri: rétention et libération);

(iii) cette frontière-filtre s'épaississant, elle est ensuite RECONNUE comme une surface d'inscription extérieure, pour d'éventuels signifiants;

(iv) enfin, lors de la phase de RÉGULATION finale, un double mouvement se produit: a) d'un côté, une règle de conversion et d'interprétation se dégage, qui associe par croisement synesthésique des signifiants-sensations avec des signifiés-matières/ énergie et les inscrit dans la «matière-mémoire» de l'enveloppe; b) de l'autre, la lumière est plongée dans une enveloppe de régénération, supposée restituer ce souvenir.

La séquence: [Contenance et Maintenance/Filtre et Masque/Surface d'inscription/Régénération] correspond strictement à la séquence canonique que nous avons établie ci-dessus, à partir des fonctions d'enveloppe telles que Didier Anzieu les a posées naguère (Fontanille, 2002 et 2003). Mais si le processus de la constitution sémiotique du paysage est homologable 
avec ce parcours des fonctions d'enveloppe, il s'inscrit du même coup à l'intérieur d'un régime temporel spécifique, celui de la mémoire des matières, des lumières et des empreintes, celui qui convertit les interactions entre matière et énergie en opérations de marquage, d'enfouissement, de désenfouissement et d'affichage d'empreintes, et qui fait de l'enveloppe sémiotique le support temporalisé des empreintes.

\section{LE PAYSAGE ET LES PASSIONS DE LA MATIÈRE-TEMPS}

Hypothèses de travail

L'étude qui précède nous permet maintenant d'aborder la question du paysage, et du rôle de ses lumières internes, armés d'un certain nombre d'hypothèses de travail.

\section{Première hypothèse:}

$d u$ visible à l'animation des matières-substrats

La sémiotique du visible est, comme nous l'avons déjà montré antérieurement (Fontanille, 1995), une sémiotique de la lumière; c'est donc dans cette perspective générale que s'inscrit la présente étude. La sémiotique de la lumière explore la syntaxe figurative du visible, très précisément en ce qu'elle est constituée des interactions entre la lumière-énergie et les matières-substrats.

Pourtant, dans le paysage, le visible n'est jamais isolé: des parfums, des contacts, des bruits accompagnent chaque figure du visible. Mais l'association de ces différentes sensations a déjà lieu dans le paysage lui-même, à travers les énergies qui l'animent, et pas seulement dans une éventuelle réception synesthésique. Et, tout comme les figures du visible renvoient à la lumière, les autres figures sensorielles renvoient à d'autres forces constitutives de l'animation des matières-substrats du paysage: par exemple, l'air qui nous effleure ou nous bouscule, et qui est perçu comme l'effet du vent, ou encore l'odeur, qui est perçue comme l'effet des mouvements intimes de transformation de la matière organique, animale ou végétale. Et c'est justement à travers ces forces et ces énergies figuratives, reçues comme des sensations de contact, que se forge l'unité polysensorielle du paysage, sous la forme de ce que nous pourrions appeler globalement l'animation des matières-substrats.
L'animation des matières-substrats équivaut à un processus d'actantialisation de ces mêmes matières, ce qui, sous certaines conditions, revient à les traiter comme des corps-actants, dotés d'une structure matérielle dynamique et d'une enveloppe qui, sous la pression des forces extérieures et intérieures (celles, justement, de l'animation), est susceptible de remplir plusieurs rôles, dont, notamment, les rôles de contenant, de filtre ou de surface d'inscription.

\section{Deuxième hypothèse:}

la stabilisation iconique des valeurs

La seconde hypothèse concerne les diverses modalités d'agencement des modes sensoriels: dans leur rencontre avec les matières-substrats, la lumière et le visible croisent d'autres phénomènes sensoriels: le tactile, l'audible, l'olfactif, et ce sont ces croisements qui vont produire des «icônes» sensorielles, et plus précisément des «signes» constitués par la réunion d'un plan du contenu avec un plan de l'expression. Autrement dit, la signification des phénomènes sensoriels, et notamment des phénomènes lumineux du visible, n'advient que dans et par les alliances polysensorielles, y compris celles liées aux mouvements et aux motions intimes.

Comme nous l'avons montré dans Les Fleurs $d u$ $\mathrm{Mal}$, ce ne sont ni les figures lumineuses ni les figures de l'eau en mouvement qui engendrent des valeurs figuratives, mais leur interaction, et la valorisation porte très précisément sur la forme et le type de leur interaction: la lumière voilée par une eau diffuse et en suspension, la lumière lavée par immersion dans l'eau, etc.; en outre, chaque icône axiologisée sollicite une association préférentielle soit avec l'olfaction, soit avec la sensori-motricité, soit avec l'ouie. Ce qui se stabilise donc au moment de l'iconisation des valeurs, ce sont des modalités particulières d'interactions sensorielles, ainsi que des compositions polysensorielles spécifiques.

De la même manière, la valeur d'une sensation olfactive qui nous parvient dans un paysage change en fonction de ces deux mêmes paramètres: elle peut être véhiculée à distance par le vent, et diffusée soit de manière rayonnante, soit de manière 
unidirectionnelle; elle peut aussi n'être accessible qu'à proximité, ou à l'occasion d'une palpation; elle peut être enfin associée à une sensation thermique générale, ou à des bruits ambiants. À chaque mode d'association et à chaque type de composition, une nouvelle valeur iconisée apparaît, et le système de valeurs figuratives du paysage consiste justement en une distribution différentielle des valeurs thymiques sur ces différents types d'icônes.

La valeur des phénomènes que nous étudions (au sens de "ce qui vaut pour", ce qui fait l'objet d'évaluations positives ou négatives) est donc déterminée par le type d'association polysensorielle et tout particulièrement par les modalités de l'interaction entre les différentes formes de l'énergie (dont la lumière) et les matières-substrats.

Ce sont donc ces principes et ces hypothèses que nous proposons de généraliser dans la perspective d'une réflexion sur la constitution sémiotique du paysage en tant que phénomène lumineux et visible.

\section{L'OBSERVATEUR, LE POINT DE VUE,} L'EXISTENCE ET L'EXPÉRIENCE

Le paysage est, ici et maintenant, un espace et un moment pour nous, et avec nous; quoi qu'on puisse dire de l'objectivité du paysage-en-soi, il reste que les valeurs ne s'actualisent que dans la rencontre des corps et dans les esthésies. L'ici et le maintenant sont partagés: il y a donc embrayage des coordonnées spatio-temporelles du lieu avec la deixis d'observation. Mais il ne peut être question de paysage que si cette condition déictique comprend en même temps sa suspension:

(i) parce que le paysage contemplé doit être aussi le cadre figuratif imaginaire (ou projeté en rétrospection ou en prospection) pour une intégration de l'observateur en tant que participant au paysage; l'observateur est donc un actant cognitif qui, potentiellement ou actuellement, doit accomplir des parcours de visiteur, de promeneur, d'habitant, etc.;

(ii) parce que le paysage s'impose par une permanence et, notamment, par rapport à sa saisie par l'observateur, par une antériorité et une postériorité qui débordent, dans le moment même de la saisie, la rencontre déictique: il ne vaut en effet, au moment et du lieu où il est saisi, que parce qu'il existe avant, après et à partir d'autres points de vue; le traitement de ces perspectives temporelles, que ce soit pour leur procurer une manifestation passionnelle (l'attente, la nostalgie, etc.) ou pour les suspendre (cf. infra, le "présent absolu " chez Jean-Paul Chavent), est une donnée essentielle du paysage comme sémiotique-objet.

Le paysage superpose donc deux configurations spatiotemporelles, l'une directement dépendante de l'observateur et l'autre indépendante. On distinguera donc respectivement le "paysage-expérience» et le "paysage-existence», indissociables et pourtant distincts.

Cette distinction, pour avoir quelque validité théorique, ne peut pas être spécifique de ce type de sémiotique-objet spécifique qu'est un paysage. De fait, nous ébauchons, avec la distinction entre existence et expérience, un modèle de sémiotique générale qui reformule et déplace, sans la remplacer, la conception traditionnelle en "plan du contenu" et "plan de l'expression", et qui, par ailleurs, vise à dépsychologiser radicalement leur interprétation restrictive en «intéroceptivité» et "extéroceptivité». Une sémiotique générale ambitieuse, en effet, ne peut plus échapper à l'ancrage ontologique de ses objets; l'existence et l'expérience constituent à cet égard les deux manières - les deux pôles d'une saisie "ontologique» par lesquelles, dans toute l'histoire de la pensée et de la philosophie, les hommes recherchent le «sens de l'être et du devenir".

La première dimension, celle de l'existence, résulte d'un débrayage ontologique: c'est au cours du passage de l'être à l'existence que l'on «tombe» dans le changement. La catégorie concernée oppose alors simplement l'existence et l'inexistence.

L'alternative résidera par conséquent dans un refus de ce débrayage ontologique: une autre conception du procès, donc embrayée, reposant sur la constance (ce qui ne varie pas dans la variation, ce qui ne s'interrompt jamais dans le changement); s'il y a structure ou système, ce ne peut être que la structure du 
changement et le système des transitions, de sorte que la constance apparaît comme une propriété du changement lui-même. L'opposition entre apparitions et disparitions a ainsi remplacé l'opposition entre existence et inexistence.

La seconde dimension, celle de l'expérience, se présente d'emblée comme «fusionnelle» et oppose à la première un embrayage ontologique. L'expérience, en effet, ne peut se concevoir sans un rapport direct avec le monde, ce qui en fait à la fois le prix et le risque (on peut se rappeler qu'une des acceptions de l'étymon experimentum est le risque, voire le danger).

L'existence ne trouve son sens que dans la disjonction, dans la distension et les différentes manières de les réduire ou de les compenser.

L'expérience, en revanche, ne trouve son sens que dans l'immersion dans le monde. C'est un processus créateur d'objets de valeur (des connaissances, des souvenirs, des compétences, des routines, des empreintes, etc.), dont le critère décisif est l'immédiateté de la relation aux objets, aux situations, au monde en général; il s'agit d'éprouver les choses, dit Le Robert, et toutes les variantes en diachronie le confirment: faire l'essai de, tenter et risquer, c'est, entre autres et d'abord, se confronter directement aux choses et aux faits. C'est pourquoi on peut définir l'expérience comme production et acquisition de valeurs grâce à l'immédiateté de la relation au monde.

Dans le cas de l'existence, tout le système axiologique prend forme à partir du débrayage ontologique (disjonction, distension, choir, déchoir, etc.), et la valence qui garantit la valeur des objets est celle de la médiation. En outre, le temps de l'existence s'impose aux matières-substrats, notamment par le biais de l'animation: de fait, les matières animées sont des matières-temps, des souvenirs et des attentes de transformations

Dans le cas de l'expérience, fondée sur l'embrayage ontologique (immersion, transition, participer, éprouver), c'est l'immédiateté qui est la valence et la garantie de la valeur des productions et acquisitions. L'immédiateté ne s'oppose pas directement à la disjonction, mais, plus fondamentalement, à la jonction, au principe même selon lequel les sujets et les

\begin{tabular}{|l|c|c|}
\hline RÉGIME ONTOLOGIQUE & EXISTENCE & EXPÉRIENCE \\
OPÉRATION FONDATRICE & Débrayage & Embrayage \\
DOMINANTE PRÉDICATIVE & Jonction & Présence \\
ÉNONCÉS TYPIQUES & Existence/Inexistence & Apparition/Disparition \\
VALENCE & Médiation & Immédiateté \\
DOMAINES & Étre, faire, & Éprouver, vivre \\
& Faits, causes et effets & Sensibles, phénomènes \\
& & \\
\hline
\end{tabular}

objets étant dissociés, leur relation ne peut être que de conjonction ou de disjonction; l'immédiateté (versus la médiation) est de l'ordre de la présence (présence au monde, en l'occurrence). Ces quelques propositions peuvent être résumées par le tableau ci-dessus.

L'hypothèse que nous formulons alors est la suivante:

(i) la constitution sémiotique du monde naturel résulte de la confrontation-transposition entre ces deux régimes ontologiques, et

(ii) comme ils sont déjà définis chacun par une opération spécifique (le débrayage et l'embrayage ontologiques), on peut aisément concevoir les opérations sémiotiques qui permettent de passer de l'un à l'autre (la confrontation-transposition) comme des embrayages et des débrayages;

(iii) l'embrayage ontologique (du régime de l'existence vers celui de l'expérience) produit un "plan de l'expression"; le débrayage ontologique (du régime de l'expérience vers celui de l'existence) produit un "plan $d u$ contenu»: ainsi se constitue une sémiotique-objet à partir du monde naturel;

(iv) les transpositions de l'existence en expérience et réciproquement affectent un très grand nombre de propriétés et catégories sémiotiques: elles expliquent notamment pourquoi une des opérations liminaires du processus sémiotique est une "prise de position» (le choix d'un "point de vue»).

Revenons maintenant au paysage.

Le "paysage-existence" est au sens strict un "pays", un lieu d'accueil, un contenant pour des contenus, ou en attente de contenus; le "paysage-expérience» est en revanche une construction polysensorielle, où la sensori-motricité fait le lien entre toutes les informations sensorielles pour leur procurer une signification dans la perspective d'une contemplation, d'une promenade, d'une activité en général. 
À la rencontre entre le paysage-existence et le paysageexpérience, se produit une homologation entre un lieu d'existence et un champ d'expérience. Le lieu a des bords, des distances, une forme globale, des occupants, etc.; le champ a un centre de référence, des horizons et une profondeur. Dès lors, le paysage, lieu d'accueil, devient un champ d'apparitions et de disparitions (ainsi peut-on apparaître dans le paysage, et en disparaître, reculer ou avancer en profondeur, atteindre le centre de référence ou rester à l'horizon).

Dans cette perspective, la composition du paysage, l'agencement de ses parties, obéit au même clivage: il offre une morphologie objective, indépendante de l'observateur, et une autre, résultant de l'expérience que ce dernier en fait; en somme, une méréologie existentielle et une méréologie expérientielle. Et c'est dans le rapport inévitable entre ces deux morphologies que les associations polysensorielles sont indispensables: la diversité sensible des matières et des surfaces du paysage-existence (notamment leurs corrélats tactiles et sensori-moteurs) est convertie en figures sensibles du paysage-expérience; les textures et les effets visuels - le mat et le brillant, le lisse et le rugueux, le modelé et le structuré, le strié et l'ondulé, etc. - sont autant de propriétés plastiques qui manifestent ces conversions sensorielles. Ce sont alors des figures qui rendent accessibles, à travers une saisie visuelle, toute la gamme des phénomènes sensoriels.

En outre, les compositions locales de traits plastiques issus de ces conversions intersensorielles permettent la reconnaissance de zones figuratives: le ciel, l'étang, la prairie, la forêt, le champ, le village, etc. Autrement dit, il nous faut faire l'expérience sensible du paysage pour accéder à ce qu'il est en tant que paysage-existence: en tant qu'expérience sensible, la différence entre l'étang et la prairie, par exemple, sous une certaine lumière, pourra se réduire à un simple contraste entre le mat et le brillant.

\section{TEMPS, MÉMOIRE}

\section{ET PRODUCTION TEMPORELLE DU PAYSAGE}

L'expérience que nous faisons du paysage ne se limite pas à la reconstruction d'une composition spatiale, et la permanence du «paysage-existence» n'est pas celle d'une maquette inerte. L'existence à laquelle nous accédons est un devenir, qui est la résultante d'une composition de processus différents: c'est ainsi, du moins, que les géographes appréhendent le paysage, comme produit d'évolutions géologiques, climatiques, économiques et culturelles.

Le paysage ne résulte pas de l'Histoire, mais d'un fourmillement d'histoires et de vitesses d'évolution différentes. Dans cette perspective, chacun des traits plastiques et chacune des figures identifiables qu'ils composent (la colline, le torrent et sa vallée, le champ, la forêt, etc.) doivent être considérés comme des «instantanés» saisis dans un mouvement. Chacune des formes identifiées est alors le signifiant d'un mouvement arrêté par la saisie; chacune des figures identifiées porte en elle, à l'état potentiel, un prédicat qui résulte de la conversion en «mouvement arrêté et instantané» d'un processus en devenir: la montagne «se dresse» dans la mesure où elle a subi une surrection; la colline s' «adoucit»: elle a été usée; la vallée s' "élargit»: la rivière l'a découpée et dégagée; le champ se "couvre" de colza jaune éclatant: l'agriculteur l'a ensemencé; les arbres sont "dépouillés»: l'automne leur a ôté leurs feuilles; le ciel est "assombri»: le vent y a poussé des nuages; etc.

On ne peut pas se contenter d'une approche rhétorique et purement verbale: ces «mouvements arrêtés" (et instantanéisés), loin d'être seulement des métaphores descriptives projetées après coup par le commentaire verbal, font partie de l'expérience que nous procure le paysage. Si rhétorique il y a, il s'agit d'une rhétorique profonde, polysensorielle et multimodale, qui configure notre expérience sensible; si de rhétorique il faut parler, alors il s'agit de la conversion des figures $d u$ paysage-existence (pris dans son devenir) en paysage-expérience (saisi comme animé de l'intérieur).

Entre ces qualificatifs de formes (les prédicats «animés» et "arrêtés») et les divers mouvements (en devenir) que nous leur associons, la saisie quotidienne et ordinaire du paysage produit une inférence purement sémiotique, en ce sens que, même si elle se présente trivialement comme une "explication", elle est avant tout la mise en relation d'un plan de 
P-existence _ P-expérience $=$ embrayage $($ production du plan de l'expression et des formes dynamiques polysensorielles)

P-expérience _ P-existence $=$ débrayage $($ production du plan du contenu et des prédicats narratifs et descriptifs)

l'expression (les propriétés dynamiques des formes: le dressé, l'élargi, l'adouci, le couvert, le dénudé, etc.) et d'un plan du contenu (des parcours thématiques, de type physique, climatique, économique ou culturel). En somme, la mise en relation du paysage-expérience avec le paysage-existence est l'opération qui constitue le paysage comme une sémiotique-objet dotée d'un plan de l'expression et d'un plan du contenu, selon les deux mouvements que nous avons définis comme embrayage et débrayage.

La notion même de "propriété dynamique des formes» mérite une explication: caractériser une forme comme "adoucie" présuppose un parcours figuratif, un régime spécifique d'interaction entre des forces et des matières, impliquant des états figuratifs virtuels comme "rugueux», «irrégulier», "régulier», "aplati», et qui, au moment même où la propriété «adoucie» est actualisée, sont potentialisés et répartis, en amont ou en aval - antérieurs ou postérieurs - de la propriété actualisée, pour former une séquence figurative. En somme, chaque prédicat descriptif du paysageexpérience fonctionne à la fois comme posé (ce qui est reconnu actuellement) et comme présupposant (ce qu'il potentialise, en amont ou en aval). Mais, là aussi, les associations polysensorielles jouent un rôle non négligeable: des nuances de vert dans une prairie, qui indiquent une composition irrégulière du sous-sol, et par conséquent une usure ou des dépôts discontinus; des stries obliques sur le flanc d'une colline aplatie, qui indiquent un très ancien plissement par surrection; un relent olfactif, qui signale une activité humaine ou une présence animale récente; ou, enfin, le bord abrupt d'un plateau, qui délimite un abîme infranchissable et signale ainsi un mouvement de faille ou d'érosion brutale. Ce sont des différences de valeur, des contrastes axiologiques, auxquels nous n'avons accès que par les propriétés plastiques du visible.

La constitution sémiotique du paysage repose en somme sur les principes suivants: 1) une confrontation-transposition entre le "paysageexistence» et le "paysage-expérience»: du premier vers le second en production, du second vers le premier en interprétation;

2) le "paysage-existence» est pris dans un devenir spatio-temporel qui procure la substance des «signifiés» du paysage (le plan du contenu); 3) le "paysage-expérience» est un lieu de conversions polysensorielles, où les propriétés phénoménales du lieu sont traduites en propriétés plastiques interprétables, et notamment en "propriétés dynamiques de formes", qui procurent la substance des «signifiants» du paysage (le plan de l'expression); 4) la réunion de ces signifiants dynamiques, plastiques et polysensoriels, avec des signifiés de «mouvements" transformateurs du paysage, produit une fonction sémiotique.

\section{N O TES}

1. Toutes les références aux Fleurs $d u$ Mal sont extraites de l'édition Gallimard, 1964, et la pagination est celle du «Livre de Poche».

2. Cette observation doit être rapprochée de la figure

d'enveloppement des corps matériels par la lumière; ce rapprochement actualise en somme la dialectique des variétés de l'enveloppe sémiotique des corps: d'un côté, la lumière procure aux corps mondains, champs, personnages ou autres, un «habit», c'est-à-dire une enveloppe de contenance et de maintenance; de l'autre, la vapeur, les larmes et la brume procurent à la source de lumière un filtre véridictoire, c'est-à-dire une enveloppe de "tri» axiologique et modal. Nous reviendrons sur ces variétés ultérieurement.

3. Le même phénomène a été observé et décrit à propos du film de Lars von Trier, Element of Crime, dans Fontanille, 1998.

4. Platon distinguait à cet égard, dans le Ménon, deux types d'âmes: celles dont la cire est suffisamment pure et de bonne texture, pour garder des empreintes nettes et durables, et celles dont la cire est impure, et la texture hétérogène, qui ne gardent que des empreintes brouillées et éphémères (ce sont les "âmes velues»). Chez Baudelaire, la membrane liquide durcie satisfait aux conditions favorables, alors que l'enveloppe poussiéreuse et sèche ne conserve aucune trace.

\section{RÉfÉREN CES BIBLIO G RAPH IQ U ES}

BAUDELAIRE, C. [1964] : Les Fleurs du Mal, Paris, Gallimard, coll. «Le Livre de Poche".

FONTANILLE, J. [1998]: «L'interprétation visuelle de l'introspection : la membrane translucide dans Element of crime (Lars von Trier) ", Protée, vol.26, no 1 , printemps;

[1999]: «Modes du sensible et syntaxe figurative ", Nouveaux Actes Sémiotiques, $\mathrm{n}^{\text {os }}$ 61-62-63, Limoges, Pulim;

[2002]: «Le corps et ses enveloppes. De la psychanalyse à la sémiotique du corps», dans Jeandillou et Esquénazi (sous la dir. de), Hommages à Michel Arrivé, Paris, L'Harmattan, coll. "Sémantiques»; __ [2003] : Séma E Soma. Figures du corps, Paris, Maisonneuve et Larose.

KANT, E. [(1798) 1979]: Anthropologie du point de vue pragmatique, trad. M. Foucault, Paris, Vrin.

ZilberberG, C. [1972]: Une lecture des Fleurs du Mal, Tours, Mame. 